\title{
The Role of Osteopontin Expression in the Prognosis of Malignant Melanoma
}

\author{
Serkan Degirmencioglu ${ }^{1 *}$, Nida Kacar ${ }^{2}$, Arzu Yaren $^{1}$ and Nese Callı Demirkan ${ }^{3}$ \\ ${ }^{1}$ Department of Medical Oncology, Pamukkale University Hospital, Turkey \\ ${ }^{2}$ Department of Medical Dermatology, Pamukkale University Hospital, Turkey \\ ${ }^{3}$ Department of Medical Pathology, Pamukkale University Hospital, Turkey \\ *Corresponding author: Serkan Degirmencioglu, Pamukkale University Hospital Fahri Goksin Oncology Center 20100 Denizli, Turkey
}

Submission: 㭗 February 10, 2018; Published: 觜 March 06, 2018

\begin{abstract}
Aim: Osteopontin is a multifunctional extracellular matrix glycoprotein that plays role in cell adhesion, migration and invasion. Increased expression of osteopontin is related to poor prognosis in various tumors. This study aims to evaluate the role of osteopontin expression in cases of metastatic and non-metastatic melanomas.

Materials and methods: A total of 26 cases of melanoma, including 13 metastatic and 13 non-metastatic cases, diagnosed in the Department of Pathology at the Pamukkale University, were included in this study. An osteopontin antibody was applied immunohistochemically to the blocks of the cases, including the primary and metastatic tumors. The degree of osteopontin expression was expressed semi-quantitatively as none/mild/ intermediate/severe, and the results were compared using parameters, such as histopathological characteristics (location, depth, cellular type, number of mitoses, number of positive lymph nodes, number of total lymph nodes and presence of ulcer), age, gender, stage, survival rate and progression-free survival, and the analysis were performed using the SPSS 16.0 package program.
\end{abstract}

Findings: Osteopontin levels demonstrated a low expression in six out of eight cases without lymph node metastasis. Osteopontin levels were found to be low in four cases in which a nevus was detected on a melanoma ground. However, no statistical significance was found since the number of cases was insufficient. Progression-free survival was 16.13+-2.5 months (95\% CI 11.1-21.09) in cases with a low osteopontin level and 41.2+-15.6 months (95\% CI 10.5-71.8) in cases with a high osteopontin level. No statistical significance was detected regarding survival.

Result: Osteopontin expression in melanoma cases can be considered a significant marker of lymph node involvement and ulcer presence on the basis of the melanoma. This study should be replicated with a large case series to investigate survival rates.

Keywords: Osteopontin; Malignant melanoma; Prognosis

Abbreviations: Ldh: Serum Lactate Dehydrogenase; Opn: Osteopontin; Sibling: Small Integrin Binding Ligand N-Linked; Os: Overall Survival; Pfs: Progression-Free Survival; Ihc: Immunohistochemical; Snp: Single Nucleotide Polymorphism; Ifn: Interferon

\section{Introduction}

Malignant melanoma is the most aggressive type of skin cancer, and its prevalence is gradually increasing worldwide [1]. Despite the significant steps taken towards understanding the mechanism of progression of melanoma, non-surgical treatment options are limited. New therapeutic targets and diagnostic tools are required for cases of malignant melanoma, considering its poor prognosis.

The prognostic parameters in malignant melanoma are tumor thickness, ulceration of the primary tumor and the level of serum lactate dehydrogenase (LDH) [2]. Increased serum LDH levels can be seen in many pathological conditions in addition to melanoma. Hence, markers that are specific for melanoma are sought.

Osteopontin (OPN) is a glycophosphoprotein that belongs to the small integrin binding ligand n-linked (SIBLING) family
[3] and is synthesized mainly by many normal cell types, such as macrophages, endothelial cells, lymphocytes and neutrophils, and also by cancer cells [4]. OPN is involved in physiological processes, such as cell adhesion, wound healing, angiogenesis and immune response, but also plays a role in autoimmune processes, inflammatory musculoskeletal diseases and cancer pathogenesis [5].

OPN, as an extracellular matrix glycoprotein, affects cancer cell adhesion, cell motion and cell survival [6], and has been reported to lead tumor progression through a cancer cell signaling pathway by increasing phosphatidylinositol-3-kinase and transcription factor nuclear factor kappa b activity [7]. OPN is over expressed in many cancer types, including malignant melanoma, and contributes to tumor progression [8]. 
The tumor stage in esophageal cancer has been reported to be directly proportional to plasma OPN levels [9]. Breast cancer cells that secrete excess OPN have been reported to make lymphovascular invasions and lymph node metastasis in the early phase [10]. Clinical studies have demonstrated a significant increase in OPN expression in melanoma samples when compared to nevus samples [11]. Osteopontin has been demonstrated to be a good tumor marker in studies, and so has been evaluated as a potential target in the control of melanoma progression [12]. However, the prognostic importance of osteopontin is yet to be established. This study aims to demonstrate the tumor marker potential of intracellular OPN expression, its relation with the metastatic potential and its prognostic significance.

\section{Materials and Methods}

In the present study, the study sample included 26 patients presented to the Medical Oncology Department of the hospital who were pathologically diagnosed with malignant melanoma and who underwent sentinel lymph node dissection (13 metastatic and 13 non-metastatic). Individuals within the age range of 20-80 years and who gave written informed consent were included in this study. Patients with a performance status of 3 or poorer and/or with brain metastasis or suspected brain metastasis, those aged 80 years or older and those who declined to sign the written informed consent form were excluded from this study.

The clinical features of the study group, such as age, gender, stage, overall survival (OS) rate and progression-free survival (PFS), as well as histopathological properties, such as the location and depth of the tumor, cell type, number of mitosis, number of positive lymph nodes, number of total lymph nodes and presence of an ulcer, were evaluated.

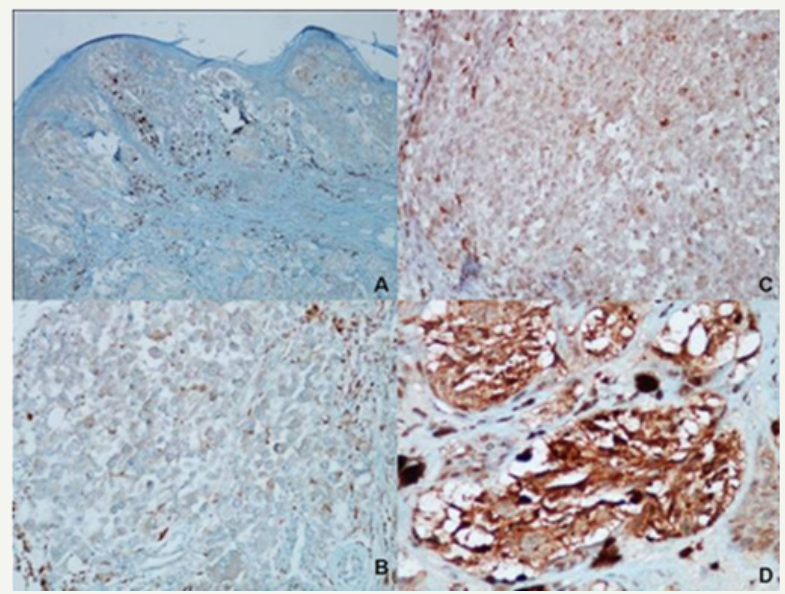

Figure 1: Osteopontin expression levels A: grade 0, B: grade $1, \mathrm{C}$ : grade 2, D: grade 3.

The immunohistochemical (IHC) OPN expression in the primary tumor and the lymph node metastasis of the tumor was evaluated. Targeted proteins became visible by dropping an OPN polyclonal antibody (dilution: 1/1000, no. ab8448; Abcam) onto the sections as a primary antibody in a fully automated IHC device (VENTANA Benchmark/LT, Ventana Medical Systems, United

States). Osteopontin expression was defined as "low" when grade 0-1 and "high" when grading 2-3 (Figure 1).

Statistical analysis was performed using the Statistical Package for Social Sciences version 16.0 (SPSS- 16.0, for Windows). The results were expressed in a 95\% t confidence interval. Statistical significance was accepted as $\mathrm{p}<0.05$. A Chi-square and a Mann Whitney-U test were used to compare the OPN expressions in various clinicopathological properties of the patients. Spearman and Pearson correlation tests were used for the correlation analysis. The Kaplan Meier method was used for the overall survival, and progression-free survival and time-survival curves, and logistic regression was applied in the analysis of the factors that affect survival and progression.

\section{Results and Discussion}

Table 1: Clinical and demographic characteristics of the patient group.

\begin{tabular}{|c|c|}
\hline Characteristics & Number /Percentage (\%) \\
\hline Gender (Female/Male) & $8(32) / 18(68)$ \\
\hline \multicolumn{2}{|c|}{ Localization } \\
\hline $\begin{array}{c}\text { Face and head/ Extremities/Trunk/ } \\
\text { other }\end{array}$ & $12(44) / 8(32) / 2(8) / 4(16)$ \\
\hline Lymph node metastasis (Yes/No) & $8(32) / 18(68)$ \\
\hline \multicolumn{2}{|c|}{ Histopathological type } \\
\hline $\begin{array}{c}\text { Nodular/acral lentiginous/superficial } \\
\text { dissemination/other }\end{array}$ & $9(36) / 2(8) / 2(8) / 13(48)$ \\
\hline Nevus (Yes/No) & $5(20) / 21(80)$ \\
\hline Ulcer (Yes/No) & $16(64) / 10(36)$ \\
\hline
\end{tabular}

The mean age of the patients in this study was identified as $49.29 \pm 19.96$ years, while the median age was found to be 46 years. The main clinical and demographic characteristics of the patients are given in Table 1.

Table 2: OPN expression levels according to mitosis, lymph node involvement, depth and age.

70

60

50

40

30

20

10

0

OPN low

OPN high

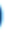

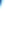

0

0

10
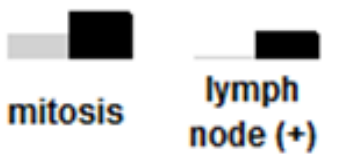

$\begin{array}{ccc}3,66 & 0,7 & 8,27 \\ 6,37 & 3,87 & 6,5\end{array}$

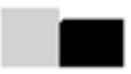

depth

age
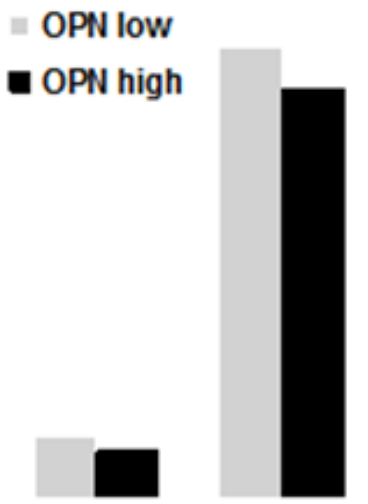

60,9

55,2 
The presence of lymph node metastasis and disease progression were statistically and significantly associated $(p=0.001)$. No difference was found between the metastatic cells in the lymph nodes and the primary tumor cells in the degree of OPN expression. Low OPN expression and high OPN expression were found in 59\% and $41 \%$ of the cases, respectively. No significant association was found between the OPN levels and age, tumor depth, lymph node involvement and the number of mitoses (Table 2).

A high degree osteopontin expression was found in six out of eight $(75 \%)$ cases with lymph node metastasis, while a low level of OPN expression was found in four cases with an ulcer in the base of the malignant melanoma. There were an insufficient number of cases to show a statistical significance. Progression-free survival in cases with a low OPN level was $6.13 \pm 2.5$ months (95\% CI 11.121.09), and $41.2 \pm 15.6$ months (95\% CI 10.5-71.8) in cases with a high OPN level ( $\mathrm{p}=0.57$ ) (Figure 2).

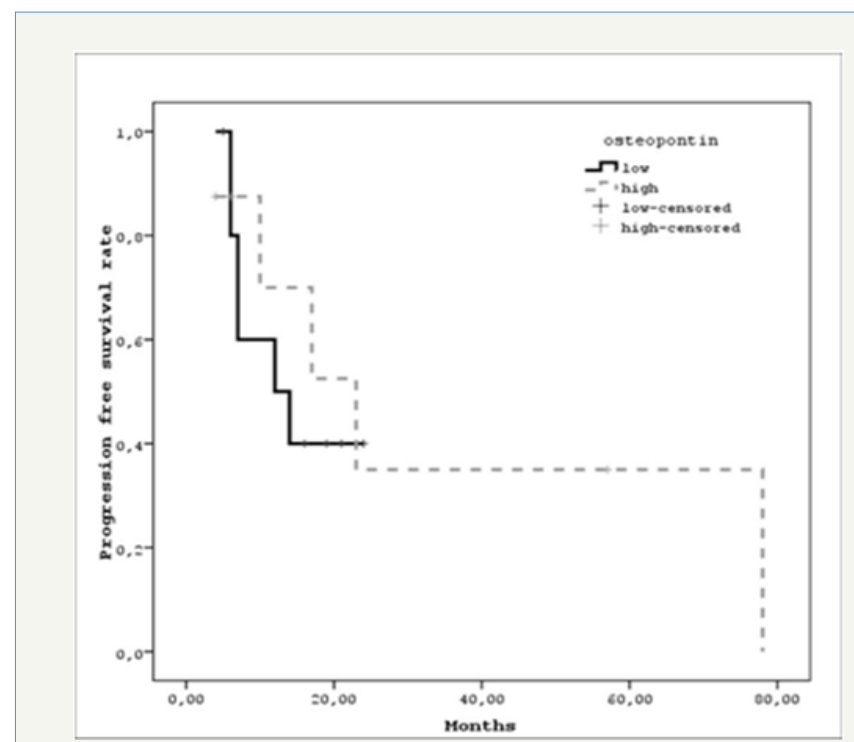

Figure 2: Kaplan Meier curve of progression-free survival according to osteopontin levels.

The importance of prognostic markers in the treatment planning and evaluation of response is gradually increasing, and immunotherapy is becoming more popular for the treatment of patients with locally advanced malignant melanoma with lymph node involvement, in addition to metastatic cases [13]. Recent studies have demonstrated the role of osteopontin in the mechanisms of cancerogenesis and metastasis [5,9].

Osteopontin was defined initially as a bone matrix protein, and was then identified as a cytokine that played role in the prognosis of cancers of the breast, lung, colon and malignant melanoma [12]. Various hypotheses have been presented regarding its role in the cancer pathogenesis. Osteopontin interacts with CD44 and epidermal growth factor receptors and causes an upregulation of metalloproteinase and urokinase plasminogen activators, and brings invasion ability to the tumor cell [6]. Osteopontin, as an apoptotic factor, causes the tumor cell to escape programmed cell death and thus causes the tumor cell to metastasize [14].
A single nucleotide polymorphism (SNP) at the 443rd position of OPN has been reported to have prognostic importance in cases of hepatocellular carcinoma and lung cancer [15-16]. The SNP stated above and the serum OPN levels are marker of response to treatment, and also for hepatitis-C cases treated with interferon [17].

A Nordic adjuvant interferon (IFN) melanoma study investigated serum OPN concentrations and OPN promotor polymorphisms in 275 high-risk malignant melanoma cases in patients receiving interferon and in patients with no therapy. The reason for evaluating osteopontin in this present study is the association of the molecule with both cancer and immune response. Interferon- $\alpha$ stimulated anti-melanoma cytotoxic $\mathrm{T}$ lymphocytes by increasing the Th1 response rate, while OPN affects Th1 cells by increasing IFN- $\alpha$ production. In the IFN melanoma study, although the survival of the cases with a high level of OPN and receiving interferon treatment was better, no statistical significance could be demonstrated [18].

High OPN expression was found to be associated with increased tumor thickness, Clark level and high mitotic index, and led to decrease PFS in previous studies. While circulation OPN levels shown no significant difference in prognosis, OPN expressiondetected IHC became an independent prognostic factor. In the same study, OPN expression was demonstrated to be a significant predictive factor for sentinel lymph node metastasis [8].

\section{Conclusion}

Osteopontin expression in cases of malignant melanoma has been considered to have a predictive role in lymph node involvement. Thus, OPN expression may be a powerful predictor of poor prognosis. The limited number of patients and the retrospective nature of this study, along with the non-standardized duration of follow-up, call for extensive multicenter prospective studies to corroborate the data obtained in this study.

\section{References}

1. Rebecca LS, Kimberly DM, Ahmedin J (2017) Cancer Statistics, 2017. CA Cancer J Clin 67(1): 7-30.

2. Balch CM, Gershenwald JE, Soong SJ (2009) Final version of 2009 AJCC melanoma staging and classification. J Clin Oncol 27(36): 6199-6206.

3. Shevde LA, Samant RS (2014) Role of osteopontin in the pathophysiology of cancer. Matrix Biol 37: 131-141.

4. Koh A, da Silva AP, Bansal AK, Bansal M, Sun C, et al. (2007) Role of osteopontin in neutrophil function. Immunology 122(4): 466-475.

5. Bandopadhyay M, Bulbule A, Butti R (2014) Osteopontin as a therapeutic target for cancer. Expert Opin Ther Targets 18(8): 883-895.

6. El-Tanani MK, Campbell FC, Kurisetty V, Jin D, McCann et al. (2006) The regulation and role of osteopontin in malignant transformation and cancer. Cytokine Growth Factor Rev 17(3): 463-474.

7. Shevde LA, Das S, ClarkDW, Samant RS (2010) Osteopontin: an effector and an effect of tumor metastasis. Curr Mol Med 10(1): 71-81.

8. Rangel J,NosratiM, Torabian S, Shaikh L, Leong SP, et al. (2008) Osteopontin as a molecular prognostic marker for melanoma. Cancer 112(1): 144-150. 
9. Lin J, Myers AL, Wang Z, Nancarrow DJ, Ferrer-Torres D, et al. (2015) Osteopontin (OPN/SPP1) isoforms collectively enhance tumor cell invasion and dissemination in esophageal adenocarcinoma. Oncotarget 6(26): 22239-22257.

10. Allan AL, George R, Vantyghem SA, Lee MW, Hodgson NC, et al. (2006) Role of the integrin-binding protein osteopontin in lymphatic metastasis of breast cancer. Am J Pathol 169(1): 233-246.

11. Smith AP, Hoek K, Becker D (2005) Whole-genome expression profiling of the melanoma progression pathway reveals marked molecular differences between nevi/melanoma in situ and advanced-stage melanomas. Cancer Biol Ther 4(9): 1018-1029.

12. Mitra A, Conway C, Walker C, Cook M, Powell B, et al. (2010) Melanoma sentinel node biopsy and prediction models for relapse and overall survival. Br J Cancer 103(8): 1229-1236.

13. Weber J, Mandala M, Del Vecchio M, Gogas HJ, Arance AM, et al. (2017) CheckMate 238 Collaborators. Adjuvant Nivolumab versus Ipilimumab in Resected Stage III or IV Melanoma. N Engl J Med (19): 1824-1835.

Creative Commons Attribution 4.0

International License

For possible submission use the below is the URL
14. Hsieh YH, Juliana MM, Hicks PH, Feng G, Elmets C, et al. (2006) Papilloma development is delayed in osteopontin-null mice: implicating an antiapoptosis role for osteopontin. Cancer Res 66(14): 7119-7127.

15. Dong QZ, Zhang XF, Zhao Y (2013) Osteopontin promoter polymorphisms at locus -443 significantly affect the metastasis and prognosis of human hepatocellular carcinoma. Hepatology 57: 1024-1034.

16. Chen Y, Liu H, Wu W, Li Y, Li J (2013) Osteopontin genetic variants are associated with overall survival in advanced non-small cell lung cancer patients and bone metastasis. J Exp Clin Cancer Res 32: 45.

17. Naito M, Matsui A, Inao M (2005) SNPs in the promoter region of the osteopontin gene as a marker predicting the efficacy of interferon-based therapies in patients with chronic hepatitis C. J Gastroenterol 40(4): 381-388.

18. Prasmickaite L, Berge G, Bettum IJ, Aamdal S, Hansson J, et al. (2015) Evaluation of serum osteopontin level and gene polymorphism as biomarkers: analyses from the Nordic Adjuvant Interferon alpha Melanoma trial. Cancer Immunol Immunother 64(6): 769-776.

\section{Your subsequent submission with Crimson Publishers} will attain the below benefits

- High-level peer review and editorial services

- Freely accessible online immediately upon publication

- Authors retain the copyright to their work

- Licensing it under a Creative Commons license

- Visibility through different online platforms

- Global attainment for your research

- Article availability in different formats (Pdf, E-pub, Full Text)

- Endless customer service

- Reasonable Membership services

- Reprints availability upon request

- One step article tracking system 